\title{
L-H transition in the presence of magnetic perturbations in ASDEX Upgrade
}

\author{
F. Ryter, S.K. Rathgeber, E. Viezzer, W. Suttrop, A. Burckhart, \\ R. Fischer, B. Kurzan, S. Potzel, T. Pütterich \\ and the ASDEX Upgrade Team \\ Max-Planck-Institut für Plasmaphysik, EURATOM Association, D-85748 Garching \\ E-mail: ryter@ipp.mpg • de
}

\begin{abstract}
The L-H transition and the H-mode behaviour in the presence of non-axisymetric $\mathrm{n}=2$ magnetic perturbations have been investigated. At low density no effect on the L-H transition is observed. Within a rather narrow density window around $50 \%$ of the Greenwald density limit, a transition to H-mode with small ELMs only and good confinement can be achieved. However, a strong density dependence of the L-H threshold power in the presence of magnetic perturbations forces the plasmas to remain in L-mode when the density is above $60 \%$ of the Greenwald value. The H-mode confinement time is not affected by the presence of the magnetic perturbations. All these H-modes, with and without ELM mitigation, exhibit a common confinement degradation with increasing recycling.
\end{abstract}

\section{Introduction}

As the power load released by large ELMs to the divertor will not be acceptable in ITER, during the recent years mitigation techniques have been investigated in several tokamaks. One promising method is offered by applying non-axisymetric magnetic field perturbations, labelled here MPs, using adequate saddle coils. This indeed leads to plasmas in which the H-mode can be kept with very small or even completely suppressed ELMs, [1, 2, 3, 4, 5]. The ASDEX Upgrade tokamak has recently been equipped with such saddle coils, labelled 
B-coils and described in [6]. In particular, they can produce an $n=2$ perturbation, where $n$ is the toroidal mode number. Applying this configuration reduces the amplitude of the ELM crashes, as well as the power load to the divertor, by at least one order of magnitude [7]. A specific feature of the ELM mitigation in ASDEX Upgrade is that it occurs at rather high plasma density, above a value labelled $n_{e, m i t i g}$, which corresponds typically to $70 \%$ of $n_{G W}$, the Greenwald density limit [8]. The value of $n_{e, m i t i g}$ indeed increases with plasma current as $n_{G W}$. The mitigation does not depend on whether the perturbation is resonant or not. The high density condition for mitigation is also reflected in the effects on the L-H transition as shown in the present paper.

As accessing the H-mode with the foreseen heating power will be crucial in ITER, it is essential to investigate the effect of the magnetic perturbations on the $\mathrm{L}-\mathrm{H}$ transition and its power threshold, $P_{t h r}$. The recent results on this topic, published for different tokamaks, MAST [5], DIII-D, [9], NSTX, [10], can be summarised as follows. These studies have all been carried out with an $n=3$ setting for the MPs, whereas our studies have been carried out with $\mathrm{n}=2$. They all indicate an increase of $P_{t h r}$ with the amplitude of the applied perturbation, which can reach up to a factor of 2 above the value without MPs. Below a certain amplitude of the perturbation field, the L-H transition is unaffected. These experiments have been carried out at medium density compared to $n_{G W}$ and no density dependence is explicitly mentioned, in contrast to our results, as will be shown below.

The goals of the ASDEX Upgrade experiments presented here were to achieve transitions to H-mode without a single large ELM, i.e. full avoidance of type-I ELMs, and to assess the effect of the MPs on the power threshold. The paper is structured as follows. The experimental procedure is described in section 2. The effect of the MPs on the characteristics of the L-H transition and ELMs are described in section 3. Power threshold and confinement properties are presented in section 4 and finally the results are discussed section 5. 


\section{Experimental approach}

As ELM mitigation in ASDEX Upgrade occurs above a threshold density, one may anticipate that a transition to H-mode followed by small ELMs only in the presence of MPs will also require a minimum density. Indeed, we find that at low density the MPs have no effect on the L-H transition, whereas at higher density L-H transitions followed by type-III ELMs only are achieved. Increasing density further prevents the plasma from transitioning into H-mode, the plasma remains in L-mode up to high heating power. Therefore, we designed the discharges such that the L-H transition would occur at a density which can be varied. This, however, is constrained by two limits in the density-power diagram:

- the L-mode density limit and associated disruption which must be avoided;

- the H-mode power threshold which should be crossed at the desired density value and not below.

In practice, density and heating power must be increased simultaneously, remaining between these two boundaries, as sketched in figure 1. The density limit can be increased, to a certain extent, by applying some heating power which, however, should not be too high to avoid inducing an L-H transition at a density lower than the desired value.

In our experiments the MPs were turned on well before increasing heating power and density. This pre-emptive approach, which is not necessarily the best choice for ITER, was the only practicable way for our experiments. The option which would consist in applying the MPs just after the L-H transition, thus mitigating the ELMs but not influencing the L-H threshold, will be discussed in the last section.

The experiments presented here were carried out in the usual parameter ranges of ASDEX Upgrade, also used in [7]: plasma current of $1 \mathrm{MA}$, magnetic field value of $B_{T}=2.5 T$ corresponding to $q_{95} \approx 4.5$, in our standard divertor plasma shape with low triangularity. The ion $\nabla B_{T}$ drift was directed toward the X-point, ensuring the usual low power threshold conditions. These deuterium plasmas were heated mainly with NBI and the power ramp was achieved through power modulation with varying duty cycle, a standard procedure in 


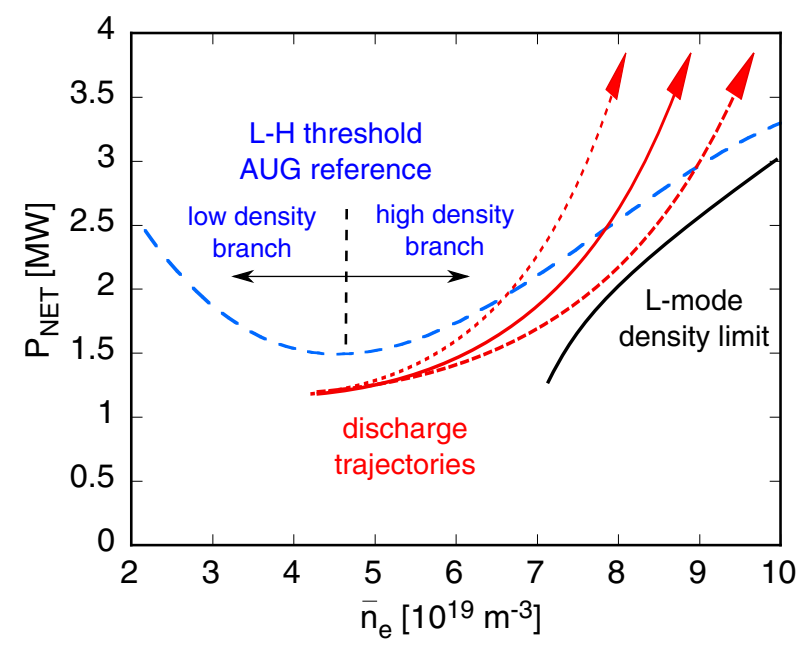

Figure 1. Operation diagram of heating power versus line averaged density. The reference $L-H$ power threshold is a fit to experimental results from [11]. The density limit is schematically indicated, as well as the required discharge trajectories according to the requirements discussed in the text.

ASDEX Upgrade and other tokamaks to investigate the L-H transition. The net heating power is defined as usual in L-H transition studies as $P_{N E T}=P_{\text {heat }}-d W / d t$ where $W$ is the plasma energy and $P_{\text {heat }}$ includes Ohmic and auxiliary heating powers for which possible losses are taken into account. As reference for the threshold power we use the ITER scaling [12] multiplied by 0.75 because this fits best the ASDEX Upgrade threshold data since 2008, [11]. This is only valid above the density minimum but applicable to the experiments of the present work which were all carried out above it. In the following we label this reference scaling for ASDEX Upgrade $P_{t h r, A U G}$. Before 2008, the power threshold was in good agreement with the ITER scaling and the recent threshold reduction might be due to the tungsten wall, but we have no direct proof for this, except the correlation in time.

The experimental operational diagram, $P_{N E T}$ versus line-averaged density is shown in figure 2 where H-mode power threshold and L-mode density limit for the parameters of the discharges used in the present work are indicated. For the density limit cases, the data are taken just before the MARFE onset because the density measurement, in particular, is strongly perturbed by the MARFE. Two discharge trajectories are also drawn which represent two different cases, 


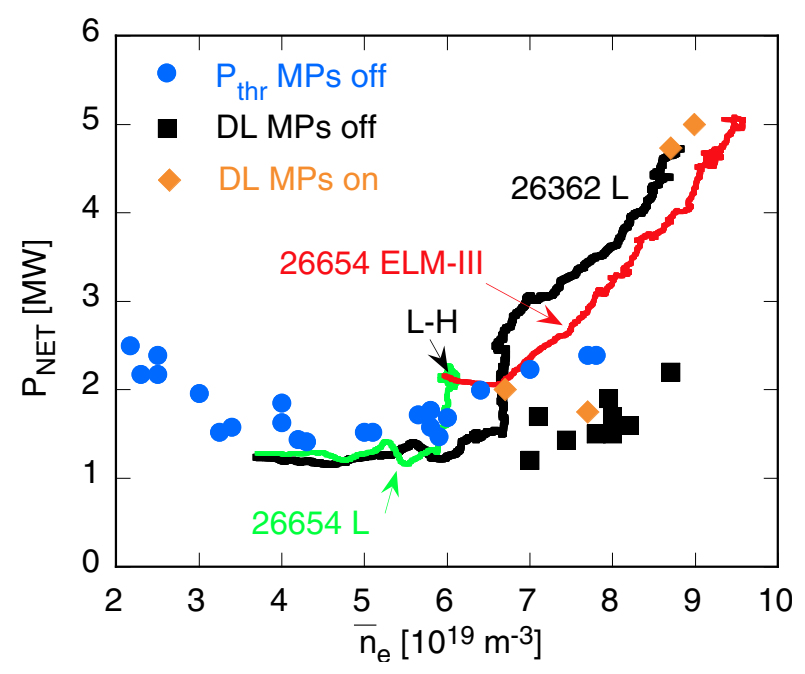

Figure 2. Net heating power versus line averaged density. The symbols specified in the legend correspond to the H-mode power threshold $P_{t h r}$ and the L-mode density limits (DL) when MPs are turned off, as well as DL points when MPs are turned on. Trajectories of two discharges for MPs turned on are also plotted and labelled as described in the text.

both with MPs turned on:

- One discharge with L-H transition to type-III ELMs (shot 26654). The transition from L to type-III H-mode is indicated in the figure. The density increase at constant power after the $\mathrm{L}-\mathrm{H}$ transition is characteristic of the $\mathrm{H}$-mode;

- One discharge which was forced to remain in L-mode by the presence of the MPs (shot 26362) and eventually reached the L-mode density limit (DL) at high heating power.

For the reasons indicated above, the discharge trajectories must remain between the $P_{t h r}$ and DL limits up to the desired density, where the L-H transition should occur. This is nicely illustrated in this figure which also shows how narrow this corridor is. It should be underlined that, under our experimental conditions at least, the density limit was reduced by about $10 \%$ by the presence of the MPs, which further reduces the operational margins. Note that the L-mode discharge 26362 was obviously very close to the density limit in the region around $\bar{n}_{e} \approx 6.5 \times 10^{19} \mathrm{~m}^{-3}$ and $P_{N E T} \approx 2 \mathrm{MW}$. If the plasma remains in L-mode when the MPs are turned on, such discharges also end with a density limit at high power and shown by the two 
diamonds in the upper right corner. In contrast, the discharges which make it into the type-III ELMy H-mode can easily be ramped to higher density without disrupting, because the density limit is higher in H-mode than in L-mode. For example, the highest density of the trajectory of discharge 26654 plotted in figure 2, representative of this case, corresponds to the density during its steady-state phase and does not lead to a disruption.

\section{L-H transition and ELM behaviour}

Following the procedure described in the previous section, we could induce the L-H transitions at different densities. As already indicated, at rather low densities, $\bar{n}_{e}<0.45 n_{G W}$, applying the MPs has no effect the on power threshold. This is illustrated in figure 3 where two discharges are compared with and without MPs, right and left plot respectively.

We show here in panel (a) the heating powers, in particular $P_{N E T}$, in box (b) the line integrated densities from the core and edge interferometry channels, whose geometry is given in [13]. In panel (c), the usual $D_{\alpha}$ signal in the divertor allows identifying the L-H transition and the ELMs. Panel (d) displays the instantaneous ELM frequency which is the inverse of the time interval between two consecutive ELMs. In panel (e) we plot the energy loss for each ELM deduced from the change in plasma energy, as well as the plasma energy divided by 10 . Therefore, the ELM loss points which coincide with the $W_{M H D} / 10$ curve correspond to $10 \%$ energy loss per ELM. Finally, panel (f) provides the H-factor, H98(y,2), with respect to the widely used ITER confinement scaling, [14], and the Greenwald fraction $f_{G W}$. In these two cases the L-H transition occurs at $f_{G W} \approx 0.4$, at the same value of $P_{N E T}$ which also corresponds to the value yielded by $P_{t h r, A U G}$, as revealed by the upper panel in each plot. Clearly, in this density range, the MPs have no influence on the L-H transition and its threshold. After the L-H transition the two discharges develop an ELM-free phase which induces a strong density increase. Consequently, for discharges 26154 in which the MPs are turned on, the density reaches a value which is close to $n_{e, m i t i g}$ and a few large ELMs occur before full mitigation which takes place at about 2.2s. Each of these few ELMs is not mitigated, their energy loss is large and would not be acceptable in ITER. We point out that 

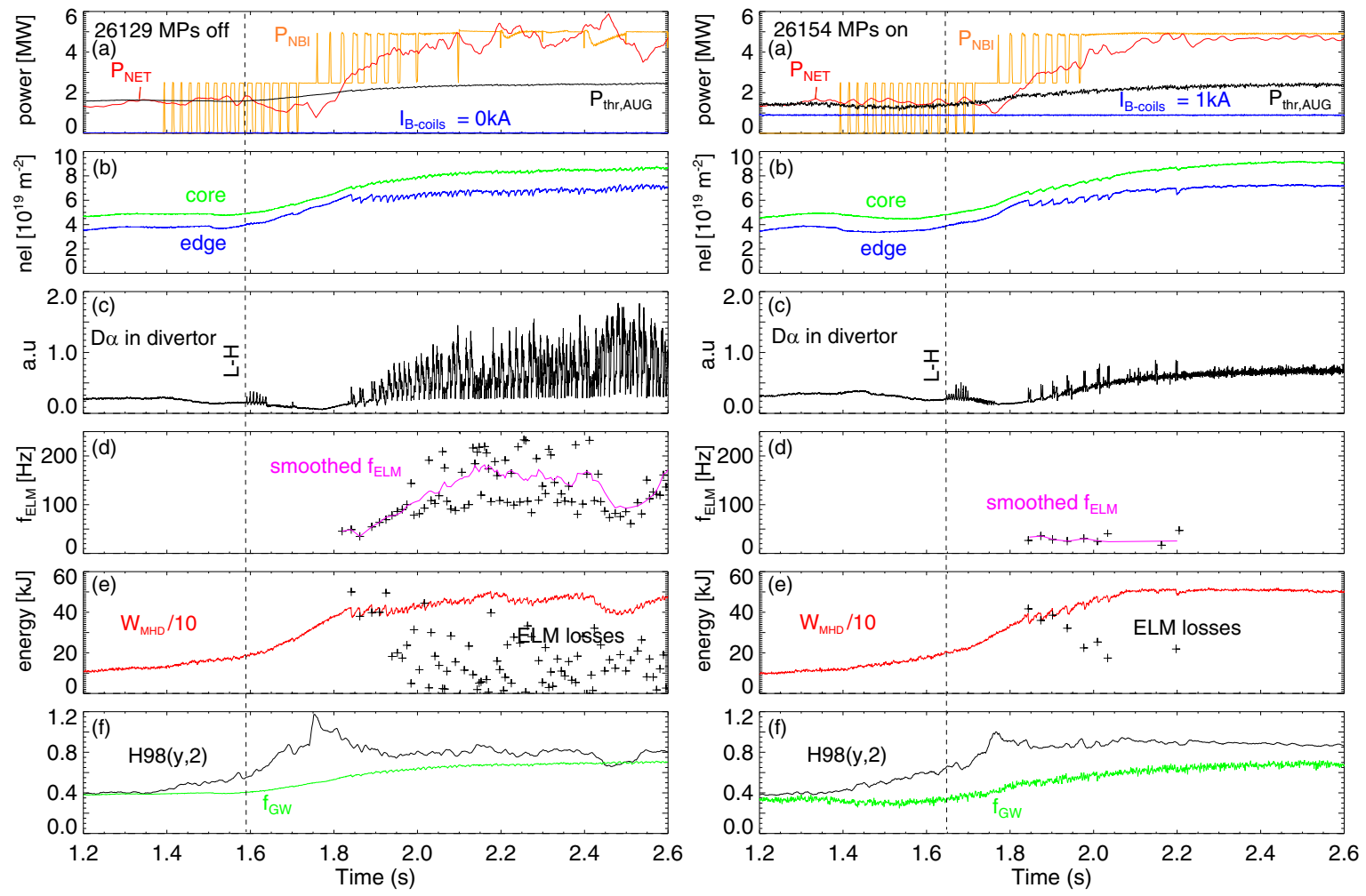

Figure 3. Time evolution of some representative quantities (indicated in the panels) comparing two discharges without MPs (left plot) and with MPs (right plot). These are low density cases where the MPs have no effect on the L-H transition, but affect the ELM behaviour as density increases after the L-H transition.

the occurrence of a few low frequency type-I ELMs during the transition to full mitigation, just below $n_{e, m i t i g}$, is a typical behaviour, [7]. Also worth noticing is that no type-III ELMs appear and that the fully mitigated H-mode is not a classical ELM-free phase, which would induce a strong density increase and end with a giant ELM, possibly inducing a transition back to L-mode. In contrast, the discharge without MPs exhibits type-I ELMs whose frequency increases with heating power and density, while the loss per ELM somewhat decreases but remains on average at a level around $5 \%$ of the plasma energy.

We conducted experiments at higher density. Before presenting them, it is worth noticing that at higher density the plasmas may exhibit some degree of detachment. This is reflected by a high level of the $D_{\alpha}$ signal in divertor which decreases as heating power is increased and has two consequences: 

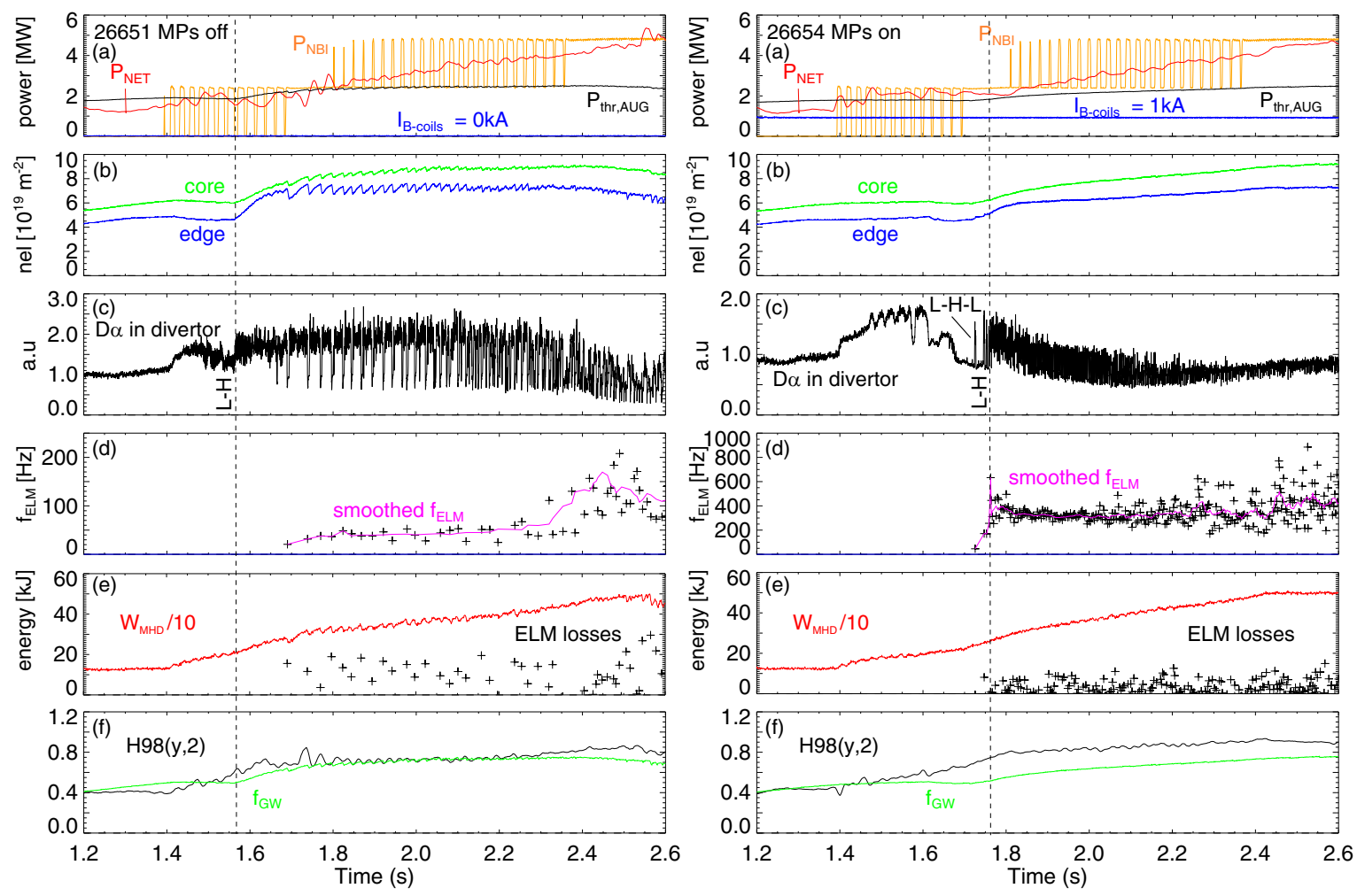

Figure 4. Time evolution of some representative quantities (indicated in the panels) comparing two discharges without MPs (left plot) and with MPs (right plot). Due to the somewhat higher density than in figure 3, the discharge with MPs on went into a type-III H-mode.

- at the L-H transition, in contrast to its familiar decrease, the $D_{\alpha}$ signal might increase due to the transiently reduced heat flux in the divertor;

- if the plasma is partially detached during the H-mode, the power load released in the divertor by each ELM reduces the detachment and induces in the $D_{\alpha}$ signal a negative spike instead of the usual positive one. This is often named ELM "burn through".

The discharges at higher density yielded the following results. At a density corresponding to $f_{G W} \approx 0.5$, the L-H transition is followed immediately by small ELMs of type-III when MPs are applied, as depicted in figure 4 right plot. In this figure, we also compare cases with and without MPs, right and left plots respectively. The two discharges transition into H-mode at very similar densities, as required, but with somewhat different heating powers. The value of $P_{N E T}$ agrees with $P_{t h r, A U G}$ for the case MPs off, whereas it is $20 \%$ higher when 
the MPs are turned on. The evolution after the L-H transition is very different in the two cases. If the MPs are turned off, the plasma, as for the cases at lower density, develops a usual ELM-free phase during which density increases strongly up to the first type-I ELM. Note the high $D_{\alpha}$ level and the ELM burn through mentioned above. The ELMs have a frequency of about $50 \mathrm{~Hz}$ and induce energy losses which reach $8 \%$ of the plasma energy content. In contrast, when the MPs are turned on, the L-H transition is followed immediately by type-III ELMs with $f_{E L M} \approx 400 \mathrm{~Hz}$ and which induce small energy losses. The few spikes on the $D_{\alpha}$ signal labelled L-H-L which occur before the L-H transition indicated by the vertical dashed line correspond to short H-mode phases which fall back into L-mode after less than $10 \mathrm{~ms}$. The spikes are positive due to the heat flux reduction caused by each L-H transition which transiently induces detachment, as explained above. Small ELMs with low energy losses occur in the middle of these first short H-modes.

In these two cases with and without MPs, the confinement time yields $H 98(y, 2) \approx 0.8$ and is comparable to that of the discharges reported in [7] with full mitigation. The confinement properties will be discussed in more detail in section 5 .

The density window at which L-H transitions followed by type-III ELMs occur is rather narrow but the effect is reproducible. This is illustrated by another example in figure 5 which exhibits features very similar to discharge 26654. Here also, a few L-H-L phases occur before the final L-H transition, as indicated in the plot. The frequency of the following ELMs is somewhat higher than in the above case and the energy losses are also small.

As we attempted to increase the density, the L-H transition was prevented by applying the MPs and the plasma remained in L-mode up to values of $P_{N E T}$ two times higher than $P_{t h r, A U G}$. This is illustrated in figure 6 for shot 26652 which is compared to the above discharge 26654 which transitioned to type-III ELMs. At the time of the L-H transition of 26654, the density of 26652 is somewhat higher while heating powers and plasma energy are almost identical. After the L-H transition time of 26654, the two discharges evolve very differently: 26652 remains clearly in the L-mode as indicated by the lower confinement time. Density is increased by gas puffing and, due to the lack of a temperature pedestal, the heating power is somewhat higher 

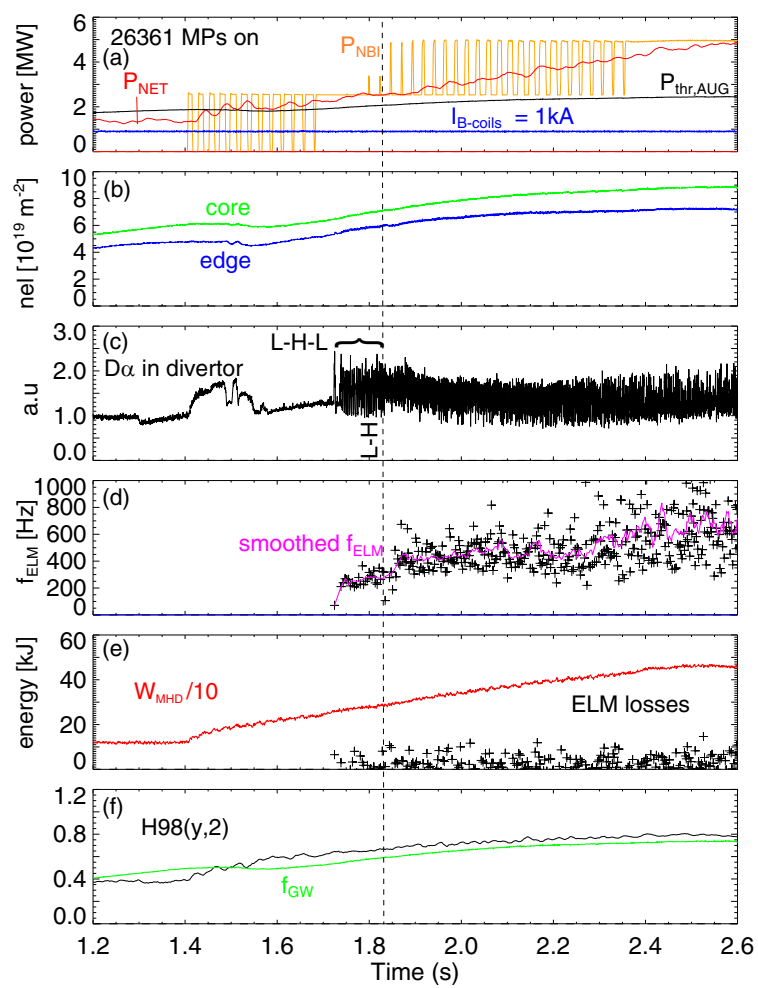

Figure 5. Time evolution of a discharge with transition into a type-III H-mode for comparison with 26654 of figure 4 right plot.
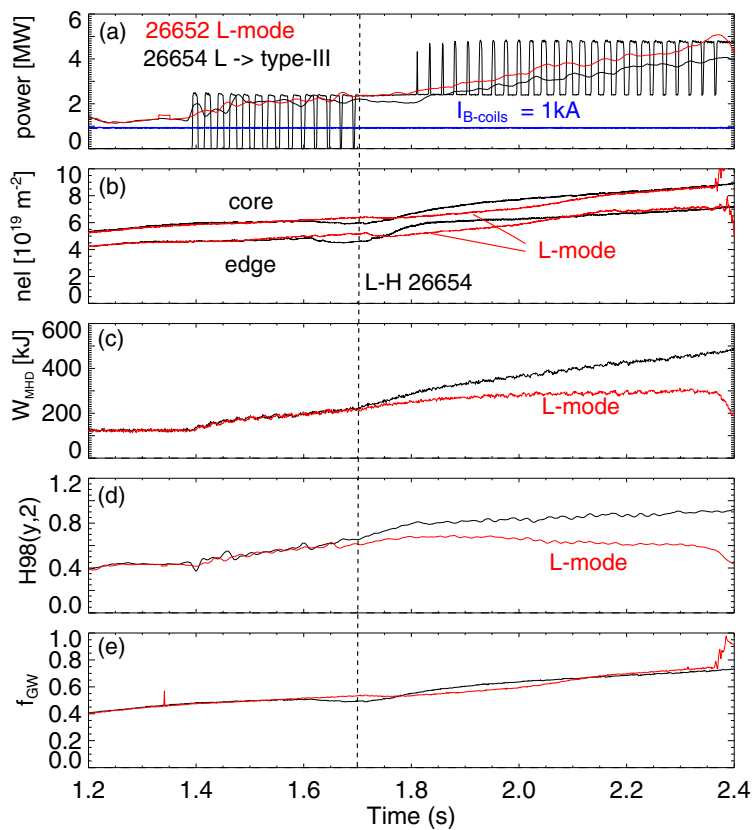

Figure 6. Time evolution of a discharge forced to remain in L-mode by the presence of the MPs (26552) and, for comparison, that of 26654 which went into a type-III $H$-mode. Same quantities as in the previous figures, with the exception of ELM data. 


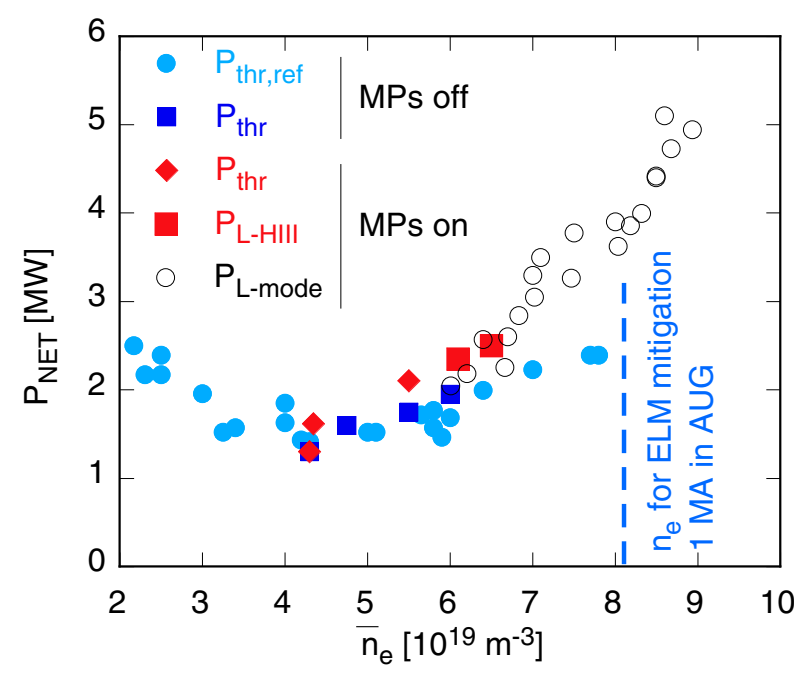

Figure 7. Net heating power versus line averaged density for the different cases indicated in the legend.

Cases without MPs: $P_{\text {thr,ref }}$ from reference L-H transition studies 2008 to 2011, mainly data from [11], $P_{\text {thr }}$ squares correspond to the 2011 series discussed in the present work. All are transitions to type-I ELMy H-modes.

Cases with MPs: $P_{\text {thr }}$ transition to type-I ELM H-modes, $P_{L-I I I}$ for transition to typeIII ELMs, $P_{L-m o d e}$ some points of the discharges forced in L-mode by the MPs.

through the Ohmic contribution and the lower $d W / d t$ term. Even at the highest heating power of about $5 \mathrm{MW}$, the discharge does not transition into the H-mode and an L-mode density limit occurs at about $2.5 \mathrm{~s}$, preceded by a MARFE which develops at about $2.35 \mathrm{~s}$, as can be seen on the density signals. In summary, this comparison indicates that, due to its threshold behaviour, the occurrence of the L-H transition is very sensitive to density in the presence of MPs.

\section{Power threshold and confinement}

The observations described above can be summarised in a plot representing heating power for the different cases versus density, figure 7 .

The points for $P_{t h r}$ when MPs were turned off or on are plotted. Those without MPs are provided mainly by our reference L-H threshold studies, [11], labelled in the plot $P_{\text {thr,ref }}$ and by the 2011 discharges dedicated to the work presented here. The transitions to type-III ELMs 
are specified. Some points of the discharge trajectories forced in the L-mode by the MPs are also indicated. This plot indicates clearly the absence of effect on the L-H transition at low density, as well as the slight increase of $P_{t h r}$ for the transition to type-III ELMs if density is somewhat higher and the high power cases without transition to H-mode at high density. The L-mode points provide the lower boundary of the heating power required to induce an $\mathrm{H}$-mode at this density in the presence of MPs. The actual power required to achieve an L-H transition, which is then higher than this boundary, will be one subject of the investigations foreseen for the 2012 campaign on this topic.

As the edge pressure profiles and the radial electric field well are widely recognised as important ingredients in the L-H transition physics, we attempted to relate them with the above results. For the ion data, provided by edge charge exchange spectroscopy, a reliable analysis of the edge gradient requires in ASDEX Upgrade a scan of the plasma radial position $(\approx 2 \mathrm{~cm}$ within $200 \mathrm{~ms}$ ) which could not be meaningfully performed in these discharges which evolve quickly in time. Doppler reflectometry data are also not available such that we have no reliable information on the edge radial electric field. Therefore, in this article, we are limited to data at the position of the pedestal top which we can compare in some of our cases with and without MPs. Overall, they indicate a very small influence of the MPs just before the L-H transition. This is in line with the analysis made in mitigated and non-mitigated H-modes $[15,16]$. In our cases, the ion pressure seems to be somewhat lower in the presence of MPs while the electron data vary little. This suggests that the radial electric field well is somewhat weaker in the presence of MPs, but this really requires confirmation in next experiments. For the L-mode discharges with MPs on, following the heating power and density ramps, the pedestal pressures increase above the values measured at the L-H transition in the other discharges, but the gradient and therefore the electric field well might remain below the value at the L-H transition. This also requires confirmation in future investigations. Furthermore, it should be pointed out that the L-H transition being a threshold phenomenon, very small variations, maybe not measurable, are sufficient to induce or prevent it.

Finally the confinement of these discharges is compared with usual type-I ELMy H-modes 


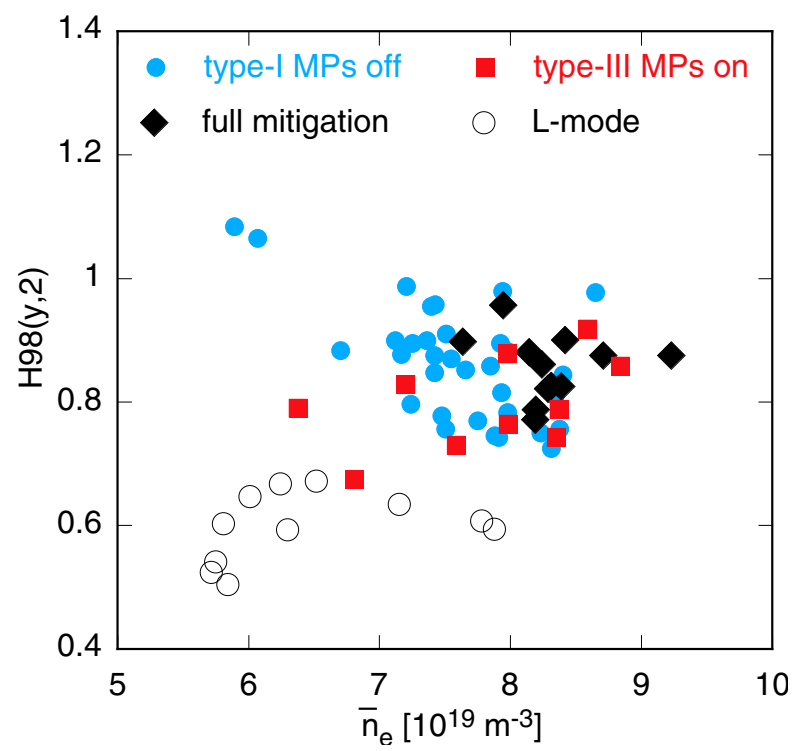

Figure 8. H-mode factor versus line averaged density for the different types of plasmas indicated in the legend. The "type-I MPs off" points are usual H-modes from the 2011 campaign.

without MPs from the 2011 campaign and with the discharges with full mitigated ELMs of the type described in [15]. Due to the relation of $n_{e, m i t i g}$ with plasma current, we consider discharges at $1 \mathrm{MA}$ only. The results are summarised in figure 8 where $\mathrm{H} 98(\mathrm{y}, 2)$ is plotted versus density. The discharges with the type-III ELMs are as good as those with fully mitigated type-I ELMy and both are within the range of the non-mitigated type-I H-modes. The L-mode points are, as expected, lower. However, this figure exhibits a rather large scatter of the H-mode points suggesting that the ITER98(y,2) scaling does not include, at least, one significant dependence.

Indeed, similarly to the results from older studies in ASDEX Upgrade, [17, 18, 19], recycling, reflected here by a measurement of the neutral gas density in the divertor region, has a significant influence, as demonstrated in figure 9. All the H-mode points are well ordered by this quantity and exhibit a clear decrease of H98(y,2). For the L-mode points the dependence is much weaker. 


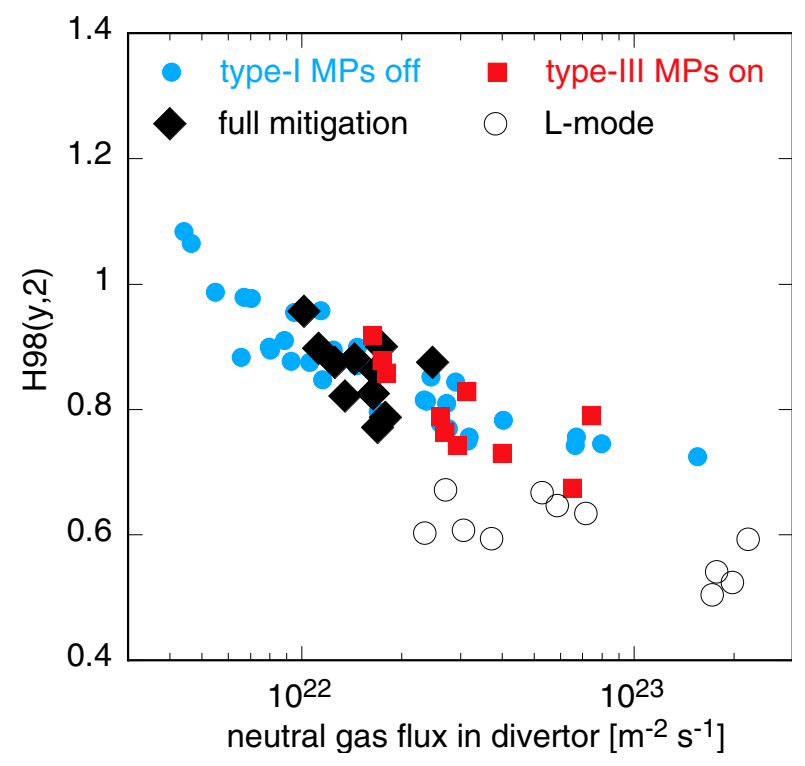

Figure 9. H-mode factor versus neutral flux measured in divertor by a gauge for the different types of plasmas indicated in the legend.

\section{Discussion}

We have shown that $\mathrm{n}=2$ magnetic perturbations do not influence the $\mathrm{L}-\mathrm{H}$ transitions below a certain density. However, as density increases, the plasma makes a transition into a $\mathrm{H}-$ mode with small ELMs of type-III which might be acceptable for ITER. When this happens, the changes of the plasma parameters induced by the L-H transition are smoother than in the usual cases which is also favourable from the plasma control point of view. Increasing density further in the presence of MPs completely inhibits the H-mode. The density window in which the transition to type-III ELMs occurs is quite narrow which requires a reliable scenario to achieve it. Further experiments might indicate ways to achieve this in a wider density range. Our experiments were carried out using the MPs in pre-emptive mode: they were turned on well before the L-H transition. One may think that turning them on just after the $\mathrm{L}-\mathrm{H}$ transition could mitigate the following ELMs without affecting the L-H transition itself. In the present situation in ASDEX Upgrade where the mitigation occurs at high density this would require to trigger the L-H transition at least at $n_{e}=n_{e, \text { mitig }}$ which is higher than what we could achieve so far in our experiments. The risk of density limit disruption would be very high, if this density 
can at all be reached. In addition, the time delay for the MPs to reach their full amplitude might also be a limiting factor.

In the 2012 campaign, ASDEX Upgrade will be equipped with 8 more B-coils, allowing us to apply MPs with toroidal mode numbers up to 4 . The mitigation results with $n=3$ achieved in the other tokamaks, $[5,9,10]$, suggest that the density dependence might be quite different, which we intend to investigate. We will also make efforts to improve the edge data which might provide more physics insight of the observed phenomena. The Doppler reflectometer is also expected to contribute data on the edge radial electric field, as well as on the influence of the MPs on turbulence and its inter-action with the GAMS.

\section{Acknowledgment}

The excellent support of the ASDEX Upgrade technical staff is warmly acknowledged. This work was partly supported by the European Communities under the contract of Association EURATOM-IPP and carried out within the framework of the EFDA Task WP10-TRA-01-03.

\section{References}

[1] HENDER, T. et al., Nuclear Fusion 32 (1992) 2091.

[2] EVANS, T. E. et al., Phys. Rev. Lett. 92 (2004) 235003.

[3] EVANS, T. E. et al., Nuclear Fusion 45 (2005) 595.

[4] LIANG, Y. et al., Phys. Rev. Lett. 98 (2007) 265004.

[5] KIRK, A. et al., Plasma Physics and Controlled Fusion 53 (2011) 065011.

[6] SUTTROP, W. et al., Fusion Engineering and Design 84 (2009) 290.

[7] SUTTROP, W. et al., Phys. Rev. Lett. 106 (2011) 225004.

[8] GREENWALD, M. et al., Nucl. Fusion 28 (1988) 2199.

[9] GOHIL, P. et al., Nuclear Fusion 51 (2011) 103020.

[10] KAYE, S. et al., Nuclear Fusion 51 (2011) 113019.

[11] RYTER, F. et al., Nuclear Fusion 49 (2009) 062003.

[12] MARTIN, Y. R. et al., Journal of Physics: Conference Series 123 (2008) 012033.

[13] MLYNEK, A. et al., Review of Scientific Instruments 81 (2010) 033507.

[14] ITER Physics Basis, Chap. II, page 2208, Table 5, Nucl. Fusion 39 (1999).

[15] SUTTROP, W. et al., Plasma Physics and Controlled Fusion 53 (2011) 124014.

[16] FISCHER, R. et al., Effect of non-axisymmetric magnetic perturbations on profiles at asdex upgrade, in 
Europhysics Conference Abstracts (CD-ROM, Proc. of the 38th EPS Conference on Plasma Physics, Strasbourg, France, 2011), edited by BECOULET, A. et al., volume 35G of ECA, page P1.072, Geneva, 2011, European Physical Society.

[17] RYTER, F. et al., Physica Scripta 51 (1995) 643.

[18] KALLENBACH, A. et al., Nuclear Fusion 35 (1995) 1231.

[19] RYTER, F. et al., Nuclear Fusion 41 (2001) 537. 